\title{
INFECÇÃO POR COVID-19 EM PACIENTES TRANSPLANTADOS DE FÍGADO
}

\section{Covid-19 infection in liver transplanted patients}

\author{
Elodie Bomfim Hyppolito 1,2,3, Beatriz Hyppolito da Justa ${ }^{3}$, Karen Soares Mendes ${ }^{3}$, Thaís Azevedo Souza Fontenele ${ }^{3}$, \\ Júlia do Carmo Barroso ${ }^{3}$, Isabela Carvalho Studart ${ }^{3}$, Lívia Caronne ${ }^{1}$, Antônio Haroldo Araújo Filho', \\ Karla Brandão Pereira1, Tarciso Daniel dos Santos da Rocha1', Cynthia Viana Ferreira Gomes¹, Clébia Azevedo', \\ Heloisa Vidal Alves Pereira', Bartolomeu Alves Feitosa Neto', Leda Fátima Rocha Miranda', Gustavo Rêgo Coelho', \\ Denissa Ferreira Gomes de Mesquita1, Paulo Ewerton Garcia', Amaury de Castro e Silva Filho', \\ Roberto da Justa Pires Neto ${ }^{1,2}$, Evelyne Santana Girão ${ }^{1,2}$, José Huygens Parente Garcia ${ }^{1}$
}

\section{RESUMO}

A infecção por SARS-Cov-2 (Covid-19) é uma condição clínica nova que impactou profundamente todo o sistema de saúde do mundo, ocasionando redução relevante na realização dos transplantes de órgãos. Desconhece-se a história natural da Covid-19 em transplantados de fígado. Objetivo: Avaliar as manifestações clínicas, o comprometimento hepático, o acesso ao Sistema de Saúde e a mortalidade da infecção por SARS-COV-2 (COVID-19) em pacientes transplantados de fígado no HUWC-UFC. Métodos: Avaliamos retrospectivamente, em estudo transversal, observacional uma série de 58 casos suspeitos de COVID-19 em pacientes transplantados de fígado pelo HUWCUFC. Destes, 18 casos tiveram confirmação de infecção por SARS-CoV-2. Resultados: Perfil epidemiológico dessa casuística: 13 indivíduos do sexo masculino, média de idade 58,8 anos ( $\Delta 35$ a 75 anos), tempo médio de transplante de fígado de cinco anos ( $\triangle 0,5$ a 11 anos). Todos os pacientes desta série de casos tinham alguma comorbidade, sendo diabetes, sobrepeso e HAS as mais frequentes. A maioria desses pacientes foi tratada em ambiente hospitalar, mas nem todos tiveram acesso à realização de tomografia de tórax, RT-PCR para SARS-CoV-2 ou à terapia intensiva, quando necessário. Dos 18 pacientes, cinco evoluíram a óbito por complicações do coronavírus, a maioria por insuficiência respiratória, implicando em uma taxa de letalidade de 27,8\%. Conclusão: Concluímos que a infecção por Covid-19 teve alta letalidade em pacientes transplantados de fígado no HUWC-UFC.

Descritores: Infecções por Coronavirus; Infecções; Transplante de Fígado; Coronavírus da Síndrome Respiratória Aguda Grave.

Instituição:

1 Univ. Federal do Ceará - Fac. Medicina - Serv. Transplante de Fígado do Hospital Universitário Walter Cantídio-Departamento de Saúde Comunitária (UFC/FAMED/DSC) - Fortaleza/CE

2 Hospital São José de Doenças Infecciosas (HSJ), da Secretaria de Saúde do Ceará - Fortaleza/CE

3 Universidade de Fortaleza - UNIFOR - Fortaleza/CE

\section{Correspondência:}

Elodie Bomfim Hyppolito

E-mail: elodie.hyppolito@gmail.com.br

Recebido em: 23/04/2021

Aceito em: 21/09/2021

\section{INTRODUÇÃO}

Em dezembro de 2019, na cidade de Wuhan, na China central, surgiram vários casos de pneumonia de etiologia até então desconhecida. Após alerta da Autoridade de Saúde da China à Organização Mundial da Saúde (OMS) sobre o ocorrido, foi identificado em janeiro de 2020, um novo coronavírus, denominado SARS-CoV-2; COVID-19 é um vírus de RNA de fita simples, moderadamente infeccioso e com mortalidade relativamente alta. 
A transmissão ocorre através das produções de gotículas respiratórias por tosse ou espirro de indivíduos infectados. ${ }^{1}$ Nos pacientes idosos, a doença tende a ser mais grave e, também, na concomitância de outras comorbidades e infecções. ${ }^{2}$

O efeito da terapia imunossupressora no COVID-19, assim como o efeito do próprio vírus em pacientes imunossuprimidos com doença hepática crônica não é totalmente compreendido. Em alguns estudos, o uso de agentes imunomoduladores para outras condições como doenças reumatológicas não resultou em aumento do risco de infecção severa por SARSCoV-2. ${ }^{3}$ À medida que o surto tornou-se uma pandemia, muitos centros de saúde em todo o mundo levantaram a preocupação de que transplantados hepáticos em terapia imunossupressora pudessem estar em alto risco de desenvolver complicações graves, casos infectados pelo novo Coronavírus, visto que esses medicamentos exercem efeitos sobre a imunidade humoral e celular e sobre a função de neutrófilos, aumentando o risco de infecções graves causadas por outros agentes virais comuns como adenovírus, rinovírus, norovírus, influenza e vírus sincicial respiratório. ${ }^{4}$

Em um estudo espanhol, a imunossupressão dos receptores detransplante defígadosemostrou controversa quanto ao prognóstico desses pacientes quando contraem o COVID-19. A elevada imunossupressão poderia ocasionar aumento da carga viral e atraso na cura dos pacientes, mas em contrapartida, o agravamento do quadro está principalmente relacionado a um sistema imune competente. ${ }^{5}$

Neste estudo, os transplantados hepáticos apresentaram incidência maior que a população geral, porém com menor mortalidade pela COVID-19 nos transplantados, sugerindo possível melhor evolução em indivíduos suprimidos. O uso de micofenolato foi associado ao aumento da mortalidade, enquanto o everolimus e inibidores de calcineurina não impactaram desfechos desfavoráveis. ${ }^{5}$ Esses dados são bem diferentes em receptores de transplante de órgão sólido hospitalizados com COVID-19 em dois grandes centros acadêmicos de Nova York, onde ocorreram taxas significativamente mais altas de doenças graves, ventilação mecânica e morte, mas se isso foi causado pelo status imunológico, pelo uso de imunossupressores ou por comorbidades subjacentes ainda não foi esclarecido. Em Bérgamo, Itália, os receptores de TOS não apresentaram desfechos diferentes da população geral. No Brasil, uma série de cinco casos de infecção ocorridos no período imediato foi descrito com a morte de dois receptores.

Assim, permanece indefinida a história natural da COVID-19 em receptores de TOS.
Segundo dados epidemiológicos publicados no site do Ministério da Saúde e da Secretaria de Saúde do Estado do Ceará, até o dia 19 de julho de 2021, o Brasil teve 210.147.125 casos notificados no e-SUS, totalizando 542.214 óbitos, e o Ceará 9.132.078 casos com 23.202 óbitos notificados. O Ceará foi um estado muito afetado pela infecção Covid-19, com expressiva morbidade e mortalidade. A pandemia de COVID-19 impactou negativamente o número de transplantes realizados, com redução de $70 \%$, no período de abril a julho de 2020.

O objetivo primário deste estudo foi avaliar as manifestações clínicas, o comprometimento hepático, - acesso ao Sistema de Saúde e a mortalidade da infecção por SARS-COV-2 (COVID-19) em pacientes transplantados de fígado no HUWC-UFC. Secundariamente, pretendemos descrever os aspectos epidemiológicos, laboratoriais e a história natural dos pacientes transplantados de fígado com COVID-19 neste serviço.

\section{MÉTODOS}

Este é um estudo transversal, quantitativo de caráter retrospectivo, do tipo documental e bibliográfico. O trabalho incluiu todos os pacientes transplantados de fígado atendidos ambulatorialmente, presencialmente ou por tele consulta, inclusive pacientes que estavam em outros estados e nos procuraram solicitando ajuda. Entre 01.03.2020 e 30.08.20, foram avaliados 58 pacientes transplantados de fígado suspeitos de COVID-19. Destes, 30 confirmaram a infecção, 18 por RT-PCR positivo para SARS-CoV-2+, 12 por testes sorológicos positivos. Foram descartados sete pacientes com RT-PCR e/ou sorologias negativas. 21 pacientes aguardavam o resultado dos testes até esta análise.

Para a análise descritiva, foram considerados casos confirmados para este estudo apenas os pacientes sintomáticos ou assintomáticos com RT-PCR+ positivo para SARS-CoV-2+, em swab de nasofaringe, por ser padrão ouro para a confirmação da infecção.

O Serviço de Transplante de Fígado do HUWCUFC estruturou durante a pandemia COVID-19 uma sala específica para triagem dos casos suspeitos de COVID-19. Todos os pacientes agendados para consulta, ao chegar ao ambulatório, passavam por uma triagem com a enfermagem, que aplicava um questionário estruturado, e em qualquer sintoma de infecção (febre, tosse, quadro gripal, diarreia, dispneia, coriza), o paciente era referido para atendimento médico em unidade de referência específica para COVID-19. Pacientes que ligassem de casa informando 
Elodie Bomfim Hyppolito,,, Beatriz Hyppolito da Justa, Karen Soares Mendes, Thaís zevedo Souza Fontenele, Júlia do Carmo Barroso, Isabela Carvalho Studart, Livia aronne, Antônio Haroldo Araújo Filho, Karla Brandão Pereira, Tarciso Daniel dos Santos da Rocha, Cynthia Viana Ferreira Gomes, Clébia Azevedo, Heloisa Vidal Alves Pereira, Bartolomeu Alves Feitosa Neto, Leda Fátima Rocha Miranda, Gustavo Rêgo Coelho, Denissa Ferreira Gomes de Mesquita, Paulo Ewerton Garcia, Amaury de Castro e Silva Filho, Roberto da Justa Pires Neto,, Evelyne Santana Girão, José Huygens Parente Garcia

que tinham sintomas suspeitos foram atendidos rotineiramente, por tele consultas por médicos e enfermeiras do serviço, mesmo que estivessem em outros estados.

Quando se identificavam casos de maior gravidade, os pacientes eram orientados a procurar as Unidades de Pronto Atendimento do seu município, para coleta do RT-PCR e para avaliação clínica presencial. A tele consulta de retorno foi realizada em todos os pacientes com suspeita de COVID-19 que o serviço tomou conhecimento. Era rotina solicitar exames de sangue, incluindo hemograma, bioquímica, gasometria arterial, raio-X de Tórax e/ou Tomografia de Tórax sem contraste (TCAR), sempre que possível.

Este estudo não realizou intervenção terapêutica específica para a infecção por coronavírus e respeitou a autonomia dos médicos e pacientes quando desejavam usar medicamentos em tratamentos compassivos para a COVID-19. O protocolo de imunossupressão do Serviço de Transplante de fígado do HUWC-UFC sofreu alguns ajustes, seguindo as orientações dos guidelines da American Association for Study of Liver Diseases 2020, European Association for the Study of the Liver 2020.

As informações foram obtidas dos prontuários dos pacientes e das tele consultas de pesquisa, e catalogadas em um questionário eletrônico no aplicativo Google Forms e analisados em planilhas do Excel Microsoft $\circledast$.

\section{RESULTADOS}

Todos os 18 casos de COVID-19 incluídos nesta série foram confirmados por RT-PCR+. Como demonstrado na tabela 1, os pacientes possuíam idade entre 35 e 75 anos, média de 58,8 anos. Treze pacientes eram homens e o tempo de transplante de fígado variou de cinco meses a 11 anos, com média de cinco anos. As causas do transplante de fígado foram Hepatite C (cinco casos), seguida por cirrose criptogênica e esteatohepatite não alcoólica com quatro casos cada, e cirrose alcoólica em três pacientes, um caso de colangite esclerosante primária (CEP) e outro de hepatite autoimune. A maioria dos casos de Covid-19 ocorreu em Fortaleza (Ceará). Todos os pacientes possuíam alguma comorbidade, sendo a diabetes a mais prevalente, seguida por sobrepeso, hipertensão arterial sistêmica (HAS) e insuficiência renal crônica (IRC). Dos 18 casos desta série, 15 foram internados, cinco evoluíram ao óbito, sendo uma em Unidade de Pronto Atendimento.
Tabela 1: Dados demográficos e perfil clínico.

\begin{tabular}{|c|c|c|c|}
\hline Variáveis & & $\mathrm{N}$ & $\%$ \\
\hline \multirow{2}{*}{ Sexo } & Masculino & 13 & 72,2 \\
\hline & Feninino & 5 & 27,8 \\
\hline Média de idade & \multicolumn{3}{|l|}{58,8 anos } \\
\hline $\begin{array}{l}\text { Média tempo } \\
\text { transplante figado }\end{array}$ & \multicolumn{3}{|l|}{5,06 anos } \\
\hline \multirow{3}{*}{ IMC } & Sobrepeso & 9 & 66,7 \\
\hline & Normal & 4 & 26,6 \\
\hline & Baixo peso & 2 & 13,3 \\
\hline \multirow{6}{*}{$\begin{array}{l}\text { Causas do transplante } \\
\text { de fígado }\end{array}$} & Hepatite C & 5 & 27,7 \\
\hline & NASH & 4 & 22,2 \\
\hline & Criptogênica & 4 & 22,2 \\
\hline & Hepatite álcoolica & 3 & 16,6 \\
\hline & CEP & 1 & 5,5 \\
\hline & Hepatite autoimune & 1 & 5,5 \\
\hline \multirow{6}{*}{$\begin{array}{l}\text { Situação especial } \\
\text { de trasplante }\end{array}$} & Hepatocarcinoma & 6 & 33,3 \\
\hline & Encefalopatia hepática & 1 & 5,5 \\
\hline & Ascite refrtária & 1 & 5,5 \\
\hline & Síndrome & 1 & 5,5 \\
\hline & Hepatopulmonar & 9 & 50,0 \\
\hline & Sem situação especial & & \\
\hline \multirow{7}{*}{$\begin{array}{l}\text { Local de notificação } \\
\text { do caso }\end{array}$} & Ceará & 8 & 44,4 \\
\hline & Maranhão & 3 & 16,6 \\
\hline & Rio Grande do Norte & 2 & 11,1 \\
\hline & Pará & 2 & 11,1 \\
\hline & Piauí & 1 & 5,5 \\
\hline & Tocantins & 1 & 5,5 \\
\hline & Não informado & 1 & 5,5 \\
\hline \multirow{4}{*}{ Óbitos por local } & Ceará & 2 & \\
\hline & Rio Grande do Norte & 1 & \\
\hline & Piauí & 1 & \\
\hline & Maranhão & 1 & \\
\hline \multirow{7}{*}{$\begin{array}{l}\text { Comorbidades/ } \\
\text { vulnerabilidades } \\
(n=18)\end{array}$} & Diabetes & 15 & 83,3 \\
\hline & Sobrepeso & 9 & 50,0 \\
\hline & HAS & 8 & 44,0 \\
\hline & $\mathrm{IRC}$ & 5 & 33,3 \\
\hline & DPOC & 1 & 5,5 \\
\hline & Cardiopatia & 1 & 5,5 \\
\hline & Retocolite ulcerativa & 1 & 5,5 \\
\hline \multicolumn{2}{|l|}{ Letalidade } & 5 & $27,7 \%$ \\
\hline
\end{tabular}

IMC: índice de massa corporal;

NASH: nonalcoholic steato hepatites;

CEP: colangite esclerosante primária;

HAS: hipertensão arterial sistêmica;

IRC: insuficiência renal crônica;

DPOC: doença pulmonar obstrutiva crônica.

De acordo com a figura 1, os sintomas mais frequentes foram febre (10), tosse (10), diarréia (9), mialgia (9), cefaleia (8), adinamia (7), anorexia (6), dispneia (6), coriza (4), anosmia (4), disgeusia (2), Odinofagia (1), Dor torácica (1), Hematoquezia (1), Dor abdominal (1) e Desmaio (1). 
Figura 1 - Sintomas dos pacientes com Covid-19

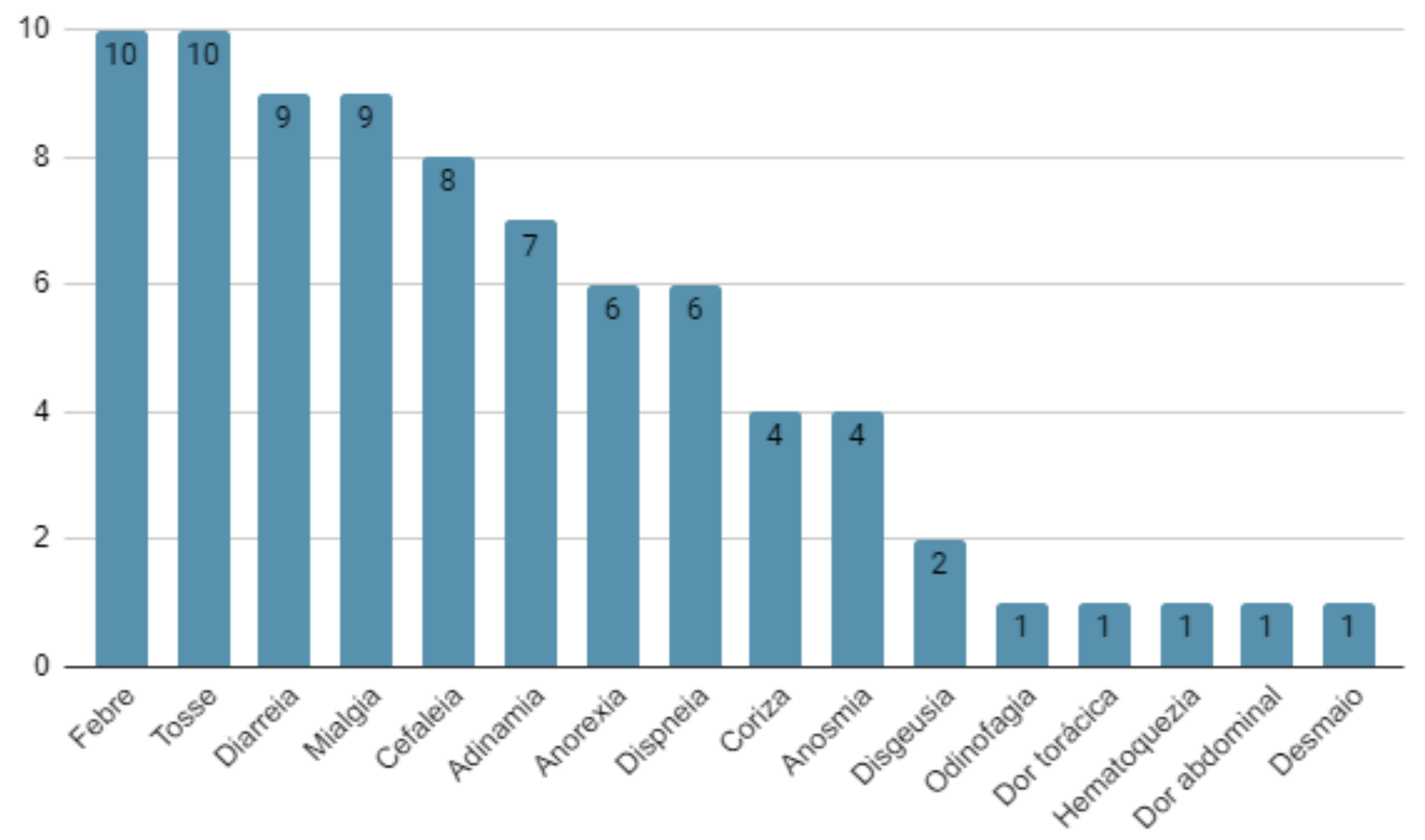

Na tabela 2, é descrito o quadro laboratorial destes pacientes. As aminotransferases estavam elevadas em dois terços dos pacientes. Quanto aos exames de função hepática, apenas um paciente apresentou elevação de bilirrubinas. Albumina e INR estavam normais nos poucos pacientes que dosaram. Linfopenia esteve presente em 55,5\% dos casos. Apenas sete pacientes realizaram TCAR na admissão.

Mais de $60 \%(n=11)$ dos pacientes investigados já realizavam imunossupressão com $F K$ e o restante realizava essa imunossupressão de forma associada $(\mathrm{FK}+\mathrm{MMF})$.

A maioria dos pacientes $(n=12)$ foi orientada a realizar redução do imunossupressor depois de diagnosticado com a COVID-19. A média do nível de FK nos pacientes foi de 6,1. Durante a fase ativa da doença, 12 pacientes foram tratados com corticoide $(n=7)$, Ivermectina $(n=6)$, Cloroquina $(n=4)$ e anticoagulante $(n=2)$.

Ainda, 16 pacientes realizaram uso de antibiótico, sendo mais de $80 \%$ a Azitromicina $(n=13)$.

Por fim, dentre os pacientes que procuraram atendimento médico, oito não precisaram de nenhum suporte ventilatório, três utilizaram o cateter nasal e dois precisaram de ventilação mecânica.
Tabela 2 - Quadro laboratorial

\begin{tabular}{|c|c|c|c|}
\hline Variáveis & & $\mathbf{N}$ & $\%$ \\
\hline \multirow{2}{*}{ AST e ALT (U/L) } & Normal & 6 & 33,4 \\
\hline & Até 3 xo normal & 12 & 66,6 \\
\hline \multirow{2}{*}{$\begin{array}{l}\text { Bilirrubina total (mg/DI) } \\
(\mathrm{n}=4 / 18)\end{array}$} & Normal & \multirow{2}{*}{$\begin{array}{l}9 \\
1\end{array}$} & \multirow{2}{*}{$\begin{array}{l}90 \\
10\end{array}$} \\
\hline & $\begin{array}{l}\text { Elevada (predomínio de } \\
\text { direta) }\end{array}$ & & \\
\hline \multirow[b]{2}{*}{ Albumina (g/DI) (n=4/18) } & Normal & \multirow[b]{2}{*}{4} & \multirow[b]{2}{*}{100} \\
\hline & $\begin{array}{l}\text { Elevada (predomínio de } \\
\text { direta) }\end{array}$ & & \\
\hline INR ( $n=4 / 18)$ & Normal & 4 & 1000 \\
\hline \multirow{3}{*}{ Linfopenia } & Presente & 5 & 27,7 \\
\hline & Ausente & 9 & 50,0 \\
\hline & Não avaliada & 4 & 22,3 \\
\hline \multirow{4}{*}{ TCAR $(n=7 / 18)$} & Normal & 1 & 14,2 \\
\hline & $\leq 25 \%$ & 2 & 28,5 \\
\hline & $26-50 \%$ & 2 & 28,5 \\
\hline & $51-75 \%$ & 2 & 28,5 \\
\hline
\end{tabular}

AST: aminotransferase de aspartate; ALT: aminotranferase de alanine; INR: indice internacional normalizado; TCAR: tomografia de tórax sem contraste. 
Elodie Bomfim Hyppolito,, Beatriz Hyppolito da Justa, Karen Soares Mendes, Thaís zevedo Souza Fontenele, Júlia do Carmo Barroso, Isabela Carvalho Studart, Livia aronne, Antônio Haroldo Araújo Filho, Karla Brandão Pereira, Tarciso Daniel dos Santos da Rocha, Cynthia Viana Ferreira Gomes, Clébia Azevedo, Heloisa Vidal Alves Pereira, Bartolomeu Alves Feitosa Neto, Leda Fátima Rocha Miranda, Gustavo Rêgo Coelho, Denissa Ferreira Gomes de Mesquita, Paulo Ewerton Garcia, Amaury de Castro e Silva Filho, Roberto da Justa Pires Neto,, Evelyne Santana Girão, José Huygens Parente Garcia

Neste estudo, também foi analisado o suporte terapêutico fornecido aos pacientes que compõem a amostra, como descrito na tabela 3. Mais de $60 \%(n=11)$ dos pacientes investigados já realizavam imunossupressão com FK, e o restante realizava essa imunossupressão de forma associada (FK + MMF). A maioria dos pacientes $(n=12)$ foi orientado a realizar redução do imunossupressor depois de diagnosticado a COVID-19. A média do nível de FK nos pacientes foi de 6,1. Durante a fase ativa da doença, 12 pacientes foram tratados com corticoide $(n=7)$, Ivermectina $(n=6)$, Cloroquina $(n=4)$ e anticoagulante $(n=2)$. Ainda, 16 pacientes realizaram uso de antibiótico, sendo mais de $80 \%$ a Azitromicina $(n=13)$. Por fim, dentre os pacientes que procuraram atendimento médico, oito não precisou de nenhum suporte ventilatório, três utilizaram o cateter nasal e dois precisaram de ventilação mecânica.

Tabela 3: Suporte terapêutico

\begin{tabular}{|c|c|c|c|}
\hline Variáveis & & $\mathbf{N}$ & $\%$ \\
\hline Imunossupressão & $\begin{array}{l}\text { FK } \\
\text { FK+MMF }\end{array}$ & $\begin{array}{c}11 \\
7\end{array}$ & $\begin{array}{l}61,1 \\
38,8\end{array}$ \\
\hline $\begin{array}{l}\text { Redução ou manutenção } \\
\text { durante a doença }\end{array}$ & $\begin{array}{l}\text { Redução da IMS } \\
\text { Manutenção da IMS } \\
\text { Suspensão do MMF }\end{array}$ & $\begin{array}{c}12 \\
3 \\
1\end{array}$ & \\
\hline Média do nível de FK & 6,1 & & \\
\hline $\begin{array}{l}\text { Tratamento compassivo } \\
\text { prescrito }(n=12 / 18)\end{array}$ & $\begin{array}{l}\text { Corticóide } \\
\text { lbermectina } \\
\text { Cloroquiina } \\
\text { Anticoagulante }\end{array}$ & $\begin{array}{l}7 \\
6 \\
4 \\
2\end{array}$ & $\begin{array}{l}58,3 \\
43,1 \\
33,3 \\
16,6\end{array}$ \\
\hline Uso de antibiótico (n=16/18) & $\begin{array}{l}\text { Azitromicina } \\
\text { Ciprofloxacina } \\
\text { Piperacilina + tazobactan } \\
\text { Vancomicina + Meropenen }\end{array}$ & $\begin{array}{c}13 \\
1 \\
1 \\
1\end{array}$ & $\begin{array}{l}81,25 \\
6,25 \\
6,25 \\
6,25\end{array}$ \\
\hline Local/tipo de atendimento & $\begin{array}{l}\text { Hospital } \\
\text { Enfermaria } \\
\text { UTI } \\
\text { Tele consulta } \\
\text { UPA }\end{array}$ & $\begin{array}{c}14 \\
10 \\
4 \\
4 \\
1\end{array}$ & $\begin{array}{l}77,7 \\
55,5 \\
22,2 \\
22,2 \\
5,55\end{array}$ \\
\hline Suporte ventilatório & $\begin{array}{l}\text { Nenhum } \\
\text { Cateter nasal } \\
\text { Ventilação mecânica }\end{array}$ & $\begin{array}{l}8 \\
3 \\
2\end{array}$ & $\begin{array}{l}44,4 \\
16,6\end{array}$ \\
\hline
\end{tabular}

FK: Tacrolimo; IMS: imunossupressão; MMF: micofenolato de sódio; UTI: unidade de terapia intensiva; UPA: unidade de pronto atendimento

\section{DISCUSSÃO}

O grande número de pacientes com suspeita de COVID-19 e o pequeno número de pacientes confirmados por RT-PCR para SARS-COV-2 refletem a grande dificuldade de acesso à realização desse teste na chamada primeira onda da COVID-19 no Ceará. $O$ perfil dos pacientes transplantados de fígado que adoeceram por COVID-19 reflete o perfil epidemiológico de pacientes acompanhados neste serviço, com predomínio do sexo masculino e faixa etária elevada. Quanto à causa do transplante de fígado, chama atenção a ausência de casos de pacientes portadores de hepatite $B$, apesar deste centro ter essa como a quarta causa de transplante de fígado. A maioria dos pacientes transplantados de fígado por hepatite $B$ no Ceará é procedente dos estados do Amazonas e Acre. Teriam esses pacientes adoecido em seus estados de origem sem que tivéssemos conhecimento ou a hepatite $B$ pode ter sido um fator de proteção à infecção pelo SARS-CoV-2? O uso crônico do entecavir que esses pacientes fazem para a prevenção de reativação póstransplante de fígado da hepatite B poderia ter sido um fator de proteção para a infecção por SARS-CoV-2 nesse perfil de pacientes?

Quanto à mortalidade, certamente temos maior confirmação dos casos mais graves e com necessidade de hospitalização implicando em mortalidade alta. A faixa etária elevada e a grande superposição de comorbidades (todos os pacientes tinham uma ou mais vulnerabilidades associadas à pior evolução clínica) certamente impactou no desfecho negativo desta série de casos. Embora a maioria tenha tido assistência médica hospitalar, nem todos os pacientes que foram a óbito tiveram acesso à terapia intensiva. Uma paciente foi a óbito em uma UPA na grande Fortaleza, após sete dias em ventilação mecânica e aguardando vaga de UTI. Em outro caso acontecido em União (PI), o paciente era portador de retocolite ulcerativa, e foi atendido em três ocasiões em Unidades Básicas de Saúde, com piora importante da diarreia na vigência da COVID-19, sendo liberado para o domicílio com saturação de $\mathrm{O} 2$ normal. Evoluiu a óbito no domicílio por causa desconhecida, não tendo sido submetido a necrópsia.

Quanto à apresentação clínica, podemos dizer que o perfil de sintomas desta série de casos é semelhante ao descrito na literatura, tendo sido a síndrome gripal, tosse, febre, mialgia e diarreia os sintomas mais comuns.

Durante a pandemia, fomos aprendendo junto com as evidências da literatura, e percebemos que o uso de tratamentos compassivos foi esporadicamente usado no início da pandemia, mas que posteriormente, o suporte ao paciente crítico, o uso de corticoide endovenoso, heparina profilática e antibioticoterapia para tratamento das infecções secundárias foram 
as únicas prescrições deste serviço. A cloroquina foi prescrita em $4 / 5$ pacientes que foram a óbito, e em apenas 2/11 que evoluíram para cura. A literatura hoje é robusta em afirmar que essa medicação está associada ao aumento da mortalidade hospitalar desses pacientes. $^{9}$ A cautela em pacientes transplantados deve ser ainda maior com tratamentos compassivos, em virtude do uso de múltiplos medicamentos ao mesmo tempo. Quanto à imunossupressão, a redução da imunossupressão foi instituída em quase todos os pacientes, mas a suspensão dos inibidores de purina foi realizada em apenas um paciente. Recentemente, a imunossupressão, incluindo os inibidores de purina, como o Micofenolato de sódio, foi um fator independente de aumento da mortalidade. ${ }^{10}$ Não observamos quadros de rejeição associados à redução da imunossupressão ou da perda do enxerto hepático neste serviço. A significativa morbidade e mortalidade da infecção por COVID-19 em pacientes transplantados de fígado serve de alerta para a necessidade de imunização desses indivíduos prioritariamente, e o máximo de isolamento possível das prováveis fontes de infecção.

\section{CONCLUSÃO}

Como conclusão deste estudo preliminar, podemos dizer que a mortalidade por SARS- COV-2 em pacientes transplantados no HUWC-UFC foi elevada. O comportamento da infecção, a gravidade e mortalidade por Sars-Cov-2 em uma série de casos consecutivamente confirmados neste serviço foi semelhante ao esperado para o perfil epidemiológico de doenças e faixa etária dos nossos pacientes. Quanto à possibilidade do uso do entecavir ter atuado como possível fator de proteção da infecção por COVID-19, são necessários estudos controlados e randomizados que possam afirmar essa hipótese aqui levantada.

\section{ABSTRACT}

SARS-Cov-2 (Covid-19) infection is a new clinical condition that has had a profound impact on the entire health system in the world, causing a significant reduction in the performance of organ transplants. The natural history of Covid-19 in liver transplant recipients is unknown. Purpose: To assess clinical manifestations, liver involvement, access to the healthcare system, and mortality of SARS-COV-2 infection (COVID-19) in liver transplanted patients at HUWC-UFC. Methods: We retrospectively assessed in a cross-sectional, observational study a series of 58 suspected cases of COVID-19 in liver transplanted patients at HUWC- UFC. Of these, 18 cases were confirmed to be SARS-CoV-2infected. Results: The epidemiological profile of this series was that 13 individuals were male, with average age of 58.8 years ( $\Delta 35$ to 75 years), mean liver transplantation time of 5 years ( $\Delta 0.5$ to 11 years). All patients in this case series had some comorbidity, with diabetes, overweight and SAH being the most frequent. Most of these patients were treated in a hospital setting, but not all had access to chest tomography, RT-PCR for SARS-CoV-2 or intensive care when necessary. Of the 18 patients, 5 patients died due to coronavirus complications, the majority due to respiratory failure, resulting in a lethality rate of $27.8 \%$. Conclusion: We conclude that Covid-19 infection had high lethality in liver transplanted patients at HUWC-UFC.

Keywords: Coronavirus Infections, Liver Transplantation, Severe Acute Respiratory Syndrome Coronavirus. 
Elodie Bomfim Hyppolito,, Beatriz Hyppolito da Justa, Karen Soares Mendes, Thaís zevedo Souza Fontenele, Júlia do Carmo Barroso, Isabela Carvalho Studart, Livia aronne, Antônio Haroldo Araújo Filho, Karla Brandão Pereira, Tarciso Daniel dos Santos da Rocha, Cynthia Viana Ferreira Gomes, Clébia Azevedo, Heloisa Vidal Alves Pereira, Bartolomeu Alves Feitosa Neto, Leda Fátima Rocha Miranda, Gustavo Rêgo Coelho, Denissa Ferreira Gomes de Mesquita, Paulo Ewerton Garcia, Amaury de Castro e Silva Filho, Roberto da Justa Pires Neto,, Evelyne Santana Girão, José Huygens Parente Garcia

\section{REFERÊNCIAS}

1. World Health Organization - 2020, Modes of transmission of virus causing COVID-19: implications for IPC precaution recommendations.

2. Harapan H, Itoh N, Yufika A, Winardi W, Keam S, Te H et al. Coronavirus disease 2019 (COVID-19): A literature review. J Infect Public Health 2020 May;13(5):667-73.

3. Kushner T; Cafardi J. Chronic Liver Disease and COVID-19: Alcohol Use Disorder/Alcohol-Associated Liver Disease, Nonalcoholic Fatty Liver Disease/Nonalcoholic Steatohepatitis, Autoimmune Liver Disease, and Compensated Cirrhosis. Clinical Liver Disease. 2020;5:195.

4. Kaltsas A, Sepkovitz K. Community acquired respiratory and gastrointestinal viral infections: challenges in the immunocompromised host. Curr Opin Infect Dis 2012;25:423-30.

5. Colmenero, Jordi, et al. "Epidemiological pattern, incidence, and outcomes of COVID-19 in liver transplant patients." Journal of hepatology 74.1 (2021): 148-155.

6. Pereira MR, Mohan S, Cohen DJ, Husain SA, Dube GK, Ratner LE et al. COVID-19 in solid organ transplant recipients: Initial report from the US epicenter. Am J Transplant. 2020;20:1800-8
7. D'Antiga L. Coronaviruses and Immunosuppressed Patients: The Facts During the Third Epidemic. Liver Transplantation, 2020;26:6.

8. Waisberg DR, Abdala E, Nasif LS, Haddad LB, Ducatti $L$, Santos VR et al. Liver transplant recipients infected with SARS-CoV-2 in the early postoperative period: Lessons from a single center in the epicenter of the pandemic. Transpl Infect Dis. 2020;4:13418.

9. Parente A, Manziat M, Angelico R, Tirotta F, Muiesan $P$, Tisone $G$ et al. (2020), COVID- 19, liver transplant and immunosuppression: allies or foes? Transpl Infect Dis. Accepted Author Manuscript. Brasil. Ministério da Saúde. Secretaria de Vigilância em Saúde. Departamento de Vigilância de Doenças e Agravos não Transmissíveis e Promoção da Saúde. Saúde Brasil 2018 uma análise de situação de saúde e das doenças e agravos crônicos: desafios e perspectivas. Brasília, Ministério da Saúde. 2020;1:1-424.

10. Cortegiani A, Ingoglia G, Ippolito M, Giarratano A, Einav S. A systematic review on the efficacy and safety of chloroquine for the treatment of COVID-19. J Crit Care. 2020 Jun;57:279-283. 